components needed for a large assembly such as a large telescope, then humans in bunny suits could perform the same sorts of extremely precise and dexterous assembly that they could be expected to perform on Earth. Calculations show that such an inflatable hangar, and the necessary gas to make it safe to occupy by shirtsleeves humans wearing oxygen masks, fits within the mass and volume limitations of the proposed "Space Launch System" heavy-lift rocket. A second launch could bring up all the com- ponents of $\mathrm{a} \approx 100$-meter-diameter or larger telescope.

A large $[200 \mathrm{ft}(\approx 61 \mathrm{~m})$ in diameter] inflated fabric sphere (or hangar) would contain four humans in bunny suits. The sphere would contain sufficient atmospheric pressure so that spacesuits would not be necessary [about 3.2 psi $\quad(\approx 22$ $\mathrm{kPa})]$. The humans would require only oxygen masks and small backpacks similar to SCUBA tanks. The oxygen content of the gas would be about $35 \%$, low enough to reduce fire risk but high enough to sustain life in the event of a failure of an oxygen mask. The bunnysuited astronauts could ride on long "cherry-picker" robots with foot restraints somewhat similar to the arm on the International Space Station. Other astronauts would maneuver freely with small propeller fans on their backpacks to provide thrust in the zero-g environment.

This work was done by Brian H. Wilcox of Caltech for NASA's Jet Propulsion Laboratory. Further information is contained in a TSP (see page 1). NPO-48441

\title{
Mars Aqueous Processing System
}

\section{This technology can be used in treating soil contaminated with heavy metals and remediation of}

acid mine drainage.

\section{Lyndon B. Johnson Space Center, Houston, Texas}

The goal of the Mars Aqueous Processing System (MAPS) is to establish a flexible process that generates multiple products that are useful for human habitation. Selectively extracting useful components into an aqueous solution, and then sequentially recovering individual constituents, can obtain a suite of refined or semi-refined products. Similarities in the bulk composition (although not necessarily of the mineralogy) of Martian and Lunar soils potentially make MAPS widely applicable. Similar process steps can be conducted on both Mars and Lunar soils while tailoring the reaction extents and recoveries to the specifics of each location.

The MAPS closed-loop process selectively extracts, and then recovers, constituents from soils using acids and bases. The emphasis on Mars involves the production of useful materials such as iron, silica, alumina, magnesia, and concrete with recovery of oxygen as a byproduct. On the Moon, similar chemistry is applied with emphasis on oxygen production.
This innovation has been demonstrated to produce high-grade materials, such as metallic iron, aluminum oxide, magnesium oxide, and calcium oxide, from lunar and Martian soil simulants. Most of the target products exhibited purities of 80 to 90 percent or more, allowing direct use for many potential applications. Up to one-fourth of the feed soil mass was converted to metal, metal oxide, and oxygen products. The soil residue contained elevated silica content, allowing for potential additional refining and extraction for recovery of materials needed for photovoltaic, semiconductor, and glass applications.

A high-grade iron oxide concentrate derived from lunar soil simulant was used to produce a metallic iron component using a novel, combined hydrogen reduction/metal sintering technique. The part was subsequently machined and found to be structurally sound. The behavior of the lunar-simulant-derived iron product was very similar to that produced using the same methods on a Michigan iron ore concentrate, which demonstrates that lunar-derived mate- rial can be used in a manner similar to conventional terrestrial iron. Metallic iron was also produced from the Mars soil simulant.

The aluminum and magnesium oxide products produced by MAPS from lunar and Mars soil simulants exhibited excellent thermal stability, and were shown to be capable of use for refractory oxide structural materials, or insulation at temperatures far in excess of what could be achieved using unrefined soils. These materials exhibited the refractory characteristics needed to support iron casting and forming operations, as well as other thermal processing needs.

Extraction residue samples contained up to 79 percent silica. Such samples were successfully fused into a glass that exhibited high light transmittance.

This work was done by Mark Berggren, Cherie Wilson, Stacy Carrera, Heather Rose, Anthony Muscatello, James Kilgore, and Robert Zubrin of Pioneer Astronautics for Johnson Space Center. Further information is contained in a TSP (see page 1). MSC-238851/4362-1 